\title{
The Interplay between Long- and Short-Range Temporal Correlations Shapes Cortex Dynamics across Vigilance States
}

\author{
Christian Meisel, ${ }^{1,2}$ Andreas Klaus, ${ }^{1}$ Vladyslav V. Vyazovskiy, ${ }^{3}$ and ${ }^{\circ D i e t m a r ~ P l e n z ~}{ }^{1}$ \\ ${ }^{1}$ Section on Critical Brain Dynamics, National Institute of Mental Health, Bethesda, Maryland 20892, ${ }^{2}$ Department of Neurology, University Clinic Carl \\ Gustav Carus, 01307 Dresden, Germany, and 32Department of Physiology, Anatomy and Genetics, University of Oxford, Parks Road, Oxford OX1 3PT, \\ United Kingdom
}

Increasing evidence suggests that cortical dynamics during wake exhibits long-range temporal correlations suitable to integrate inputs over extended periods of time to increase the signal-to-noise ratio in decision making and working memory tasks. Accordingly, sleep has been suggested as a state characterized by a breakdown of long-range correlations. However, detailed measurements of neuronal timescales that support this view have so far been lacking. Here, we show that the cortical timescales measured at the individual neuron level in freely behaving male rats change as a function of vigilance state and time awake. Although quiet wake and rapid eye movement (REM) sleep are characterized by similar, long timescales, these long timescales are abrogated in non-REM sleep. We observe that cortex dynamics exhibits rapid transitions between long-timescale states and sleep-like states governed by short timescales even during wake. This becomes particularly evident during sleep deprivation, when the interplay between these states can lead to an increasing disruption of long timescales that are restored after sleep. Experiments and modeling identify the intrusion of neuronal offline periods as a mechanism that disrupts the long timescales arising from reverberating cortical network activity. Our results provide novel mechanistic and functional links among behavioral manifestations of sleep, wake, and sleep deprivation and specific measurable changes in the network dynamics relevant for characterizing the brain's changing information-processing capabilities. They suggest a network-level function of sleep to reorganize cortical networks toward states governed by long timescales to ensure efficient information integration for the time awake.

Key words: cortical network function; sleep; sleep deprivation; timescales

Significance Statement

Lack of sleep deteriorates several key cognitive functions, yet the neuronal underpinnings of these deficits have remained elusive. Cognitive capabilities are generally believed to benefit from a neural circuit's ability to reliably integrate information. Persistent network activity characterized by long timescales may provide the basis for this integration in cortex. Here, we show that longrange temporal correlations indicated by slowly decaying autocorrelation functions in neuronal activity are dependent on vigilance states. Although wake and rapid eye movement (REM) sleep exhibit long timescales, these long-range correlations break down during non-REM sleep. Our findings thus suggest two distinct states in terms of timescale dynamics. During extended wake, the rapid switching to sleep-like states with short timescales can lead to an overall decline in cortical timescales.

\section{Introduction}

The ability of neural circuits to integrate information over extended periods of time is thought to be an essential ingredient for

\footnotetext{
Received Feb. 16, 2017; revised Aug. 7, 2017; accepted Aug. 24, 2017.

Author contributions: C.M. designed research; C.M. and A.K. performed research; C.M., V.V.V., and D.P. contributed unpublished reagents/analytic tools; C.M. analyzed data; C.M. and D.P. wrote the paper.

The authors declare no competing financial interests.

This study was supported by the Intramural Research Program of the NIMH. This study utilized the highperformance computational capabilities of the Biowulf Linux cluster at the National Institutes of Health, Bethesda, MD. V.V. is supported by Wellcome Trust Strategic Award 098461/Z/12/Z. The authors thank Chiara Cirelli and Giulio Tononi for insightful discussions.

Correspondence should be addressed to: Christian Meisel, Department of Neurology, University Clinic Carl Gustav Carus, 01307 Dresden, Germany. E-mail: christian@meisel.de.
}

information processing. In decision-making and working memory tasks (Kiebel et al., 2008; Friston et al., 2012; Chaudhuri et al., 2015; Kringelbach et al., 2015), for example, this ability may increase the signal-to-noise ratio and help to maintain some memory of past activity. The cortical networks that exhibit this ability are typically characterized by slowly decaying autocorrelation functions or, in general, long-range temporal correlations in their dynamics. Accordingly, slow autocorrelation function decays in cortical activity have been observed using different experimental modalities, including 
studies on nonhuman primates (Ogawa and Komatsu, 2010; Murray et al., 2014), human electrocorticography (Honey et al., 2012), and fMRI (Stephens et al., 2013).

Although these long timescales associated with long-range temporal correlations in the history of neural activity may provide a plausible mechanism for temporal integration during wake, their dependence on vigilance levels is currently less well understood. It has been suggested that, during sleep, the brain loses its ability to integrate information effectively across different cortical areas and across time, a functional loss that has also been proposed as a potential mechanism for the concomitant loss of consciousness (Tononi, 2008). Consistent with this hypothesis, a decrease in information integration during non-rapid eye movement (NREM) sleep has been observed in space, quantified by the effective connectivity between different cortical regions (Massimini et al., 2005) and time (Tagliazucchi et al., 2013). These studies, however, have focused on large-scale brain signals measured by EEG and fMRI; their link to the long timescales governing individual neuron activity is missing. Apart from the effect of sleep on cortical timescales, it is also unknown whether more subtle vigilance changes may affect the timescales of cortical dynamics. For example, cognitive capabilities and the ability to process information are known to be affected by extended waking (Van Dongen et al., 2003; Banks and Dinges, 2007; Mignot, 2008; Killgore, 2010). The impact of extended wakefulness on cortical timescales, however, is currently unknown.

Here, we sought to characterize systematically the timescales governing cortical dynamics during wake, extended waking, and its dependency on sleep. We made use of the notion that the network dynamics can be described in terms of their ability to integrate information over time, which can be assessed by quantifying its temporal correlations. We hypothesized that the breakdown in long-range temporal correlations during sleep will be visible at the level of individual neuron-spiking activity and that changes in temporal correlations might provide mechanistic and functional links among behavioral manifestations of sleep, waking, and sleep deprivation. For this purpose, we analyzed neuron activity at the individual and multiunit level along with local field potentials (LFPs) across different cortical regions in freely behaving rats over extended periods of time and across vigilance states.

\section{Materials and Methods}

We investigated two datasets in which cortical activity was monitored across different vigilance states in rats. In the first dataset, LFPs and multiunit activity (MUA) were recorded from frontal cortex during $6 \mathrm{~h}$ of sleep deprivation and after a consecutive recovery period ( $n=7$ animals; dataset 1). In the second dataset, LFP and single-unit activity (SUA) were recorded from frontal and parietal cortex areas during $4 \mathrm{~h}$ of sleep deprivation and during consecutive recovery sleep $(n=13$ animals; dataset 2). Dataset 1 was used for Figures 2 and 3; dataset 2 was used for Figures 1 and 2.

Dataset 1. Adult male rats (Sprague Dawley, total $n=7$ ) were used in this dataset. All rats were housed individually in transparent Plexiglas cages. Lighting and temperature $\left(21 \pm 0.5^{\circ} \mathrm{C}\right)$ were kept constant (light/ dark $12 \mathrm{~h} / 12 \mathrm{~h}$, lights on at 6:00 A.M., food and water available ad libitum).

Implantation of microelectrode arrays. Procedures were in accordance with National Institutes of Health guidelines. Animal procedures were approved by the National Institute of Mental Health Animal Care and Use Committee. At age 4-6 weeks, multichannel microelectrode arrays were implanted in the right frontal cortex (B: $+1-2 \mathrm{~mm}, \mathrm{~L}: 2.5-3.5 \mathrm{~mm}$ ) under deep isoflurane anesthesia (1.5-2\% volume) and the presence of the analgesic ketoprofen $(5 \mathrm{mg} / \mathrm{kg}, \mathrm{SC})$. Ketoprofen was given for up to $2 \mathrm{~d}$ after surgery and animals were allowed to recover for $5 \mathrm{~d}$ before recordings. The arrays had 32 channels $(8 \times 4,200 \mu \mathrm{m}$ interelectrode spacing; $23 \mu \mathrm{m}$ electrode diameter; Neuronexus). The surgical procedure was performed in sterile conditions in which, first, $\mathrm{a} \sim 2 \times 2 \mathrm{~mm}$ craniotomy was made using a high-speed surgical drill. After an incision in the dura, the electrode array was then lowered into the brain tissue by penetrating the pia mater. Electrodes were slowly advanced further into brain tissue until all rows of the array were at the level of superficial cortical layers (layers 2/3) and most channels showed robust SUA or MUA. After application of silicone gel to seal the craniotomy and to protect the surface of the brain, dental acrylic was placed around the electrode array to fix it to the skull. The ground wire was connected to a skull screw located $1 \mathrm{~mm}$ posterior to bregma.

Experimental design. At least $5 \mathrm{~d}$ were allowed for recovery after surgery. Sleep deprivation began at light onset (6:00 A.M.) and involved continuous observation of the animal and its LFP recording. Animals were sleep deprived for a total of $6 \mathrm{~h}$, after which there was an undisturbed recovery period of 5-6 h. Prolonged wakefulness was achieved by providing rats with novel objects and/or delivering low-level auditory or visual stimuli. Before the experiment, rats were habituated to the experimenter and to the exposure to novel objects (including nesting and bedding material from other rat cages, tubes of different shape and color, little colored blocks, rubber balls, and boxes of different shapes and colors). Care was taken not to stress animals; they never appeared to engage in freezing or aggressive behavior and exhibited no signs of discomfort.

LFP and MUA recording during prolonged wakefulness. Neuronal activity including LFP and extracellular unit activity was recorded at $30 \mathrm{kHz}$ and stored for offline analysis (Cerebus; Blackrock Microsystems). At least $20 \mathrm{~min}$ of continuous LFP and MUA were recorded for each hour and experiment during prolonged wakefulness [from 0 to $6 \mathrm{~h}$, corresponding to sleep deprivation 0 (SD0) to SD6, respectively], as well as after the recovery period (REC). It has been observed previously that wake is not a homogenous state and that it is therefore important to perform analyses within substates (quiet and active wake) as similarly as possible (Fisher et al., 2016). Here, similar to previous work (Vyazovskiy et al., 2011), we focused on "quiet wake" (Q) episodes; that is, periods when the animal is alert, has its eyes open, and readily responds to stimuli while maintaining vigilance and posture but is immobile. To find these episodes, we first marked periods when the animal behaviorally appeared to be in a $Q$ state: when it had its eyes open, readily responded to stimuli, maintained vigilance and posture, but was immobile. This included the removal of episodes with rearing, grooming, eating, or drinking. Channels containing artifacts or noise were removed from further analysis of LFP and MUA. We then carefully selected time periods of at least $4 \mathrm{~s}$ duration that showed LFP signals characterized by typical wake activity; that is, LFP signals characterized by mainly low-voltage activity with theta waves and no extended periods of slow waves typical for sleep $(\geq \sim 1 \mathrm{~s})$.

Similar to previous reports (Vyazovskiy et al., 2011), we observed occasional large-amplitude activity in these select periods of $\mathrm{Q}$, which typically presented as single positive half-waves in the frequency range of 2-6 Hz, but could occasionally also appear in succession of 2 or more waves (see Fig. 2c). Events like these are often not detectable in EEG (Vyazovskiy et al., 2011) and are therefore part of what is traditionally considered the wake state. To determine whether and to what extent our results depended on the occurrence of these events, all analyses were repeated on data in which segments with this kind of slow-wave activity had been eliminated (see below). As a further control, such large-amplitude segments were carefully removed from analyses in dataset 2 (see below).

Multiunit spikes were extracted from the high-pass filtered signal $(\geq 250 \mathrm{~Hz}$ ) by applying a threshold at -5 times the root mean square of the signal using Cerebus Central software (Blackrock Microsystems). Thresholded waveforms were subsequently offline sorted using the Offline Spike Sorter (Plexon) to eliminate artifactual waveforms caused by electrical or mechanical noise. Principal components (set to 3) were extracted and automatic cluster selection was performed using the T-Distribution Expectation-Maximization (E-M) method (Shoham et al., 2003). Only electrodes and units were used for analysis for which the typical biphasic waveform was evident. LFPs were first downsampled to $500 \mathrm{~Hz}$ and band-pass filtered $(1-40 \mathrm{~Hz})$ using a phase neutral filter by applying a second-order Butterworth filter in both directions. A notch filter to eliminate line noise was applied subsequently. 
Dataset 2. For dataset 2, we used recordings from a study described in detail previously (Vyazovskiy et al., 2011). In short, data consisted of male WKY rats from which LFP and unit activity were recorded in frontal $(n=11)$ and/or parietal $(n=9)$ cortex with 16-channel microarrays. Similar to dataset 1, rats were housed individually in transparent Plexiglas cages (light/dark 12/12 h, lights on at 10:00; food and water ad libitum and replaced daily at 10:00). Data recording and online spike sorting were done with the Multichannel Neurophysiology Recording and Stimulation System (Tucker-Davis Technologies). Unit activity was collected continuously $(25 \mathrm{kHz}, 300-5000 \mathrm{~Hz})$ concomitantly with the LFPs from the same electrodes and epidural EEGs (both $256 \mathrm{~Hz}, 0.1-100$ $\mathrm{Hz}$ ). Data consisted of $4 \mathrm{~h}$ of sleep deprivation and $6 \mathrm{~h}$ of consecutive recovery. In each animal, 2-4 experiments with $4 \mathrm{~h}$ of sleep deprivation were performed (at least $5 \mathrm{~d}$ apart). Sleep stages were scored offline by visual inspection of $4 \mathrm{~s}$ epochs. Spike sorting was performed by principal component analysis followed by a split and merge expectation maximization clustering algorithm. Individual unit activity was binned in $2 \mathrm{~ms}$ bins and stored for further offline analysis. All analyses in this study were performed on artifact-free $4 \mathrm{~s}$ intervals classified as vigilance stages pertaining to Q, REM sleep (R), or NREM sleep (N). Quiet wake epochs were defined as in dataset 1 (see above). In addition, $4 \mathrm{~s}$ epochs containing large-amplitude LFP activity were removed from further analysis by visual inspection, which was confirmed by the absence of a second, bimodal peak in the amplitude data (see Fig. $2 g$, blue line) in analogy to dataset 1. For further details regarding these data, see Vyazovskiy et al. (2011).

Spike count autocorrelation. The temporal autocorrelation of spike counts was computed for MUA (dataset 1) and putative single units (dataset 2) similar to previous work (Ogawa and Komatsu, 2010; Murray et al., 2014). The $4 \mathrm{~s}$ intervals were divided into separate, successive time bins of duration $\Delta$, which we set to $\Delta=100 \mathrm{~ms}$. Across different $4 \mathrm{~s}$ intervals within the same animal and hour during sleep deprivation (datasets 1 and 2), during recovery sleep (dataset 2), and after recovery sleep (dataset 1), we calculated the correlation between spike counts $N$ in two time bins, indexed by their onset times $i \Delta$ and $j \Delta$, using the Pearsons correlation coefficient $R$ as follows:

$$
R(i \Delta, j \Delta)=\frac{\operatorname{Cov}(N(i \Delta), N(j \Delta))}{\sqrt{\operatorname{Var}(N(i \Delta)) \times \operatorname{Var}(N(j \Delta))}}
$$

where covariance (Cov) and variance (Var) are computed across $4 \mathrm{~s}$ intervals for those time bins. To allow reliable computation of spike count autocorrelation, we required that each (multi-) unit fired at least 8 times during the $4 \mathrm{~s}$ interval and that at least $104 \mathrm{~s}$ intervals within each animal and hour were available (Ogawa and Komatsu, 2010); similar to previous work, this threshold level was not essential to observe our main findings. One rat of dataset 1 did not exhibit MUA at one time point during the sleep deprivation period and was thus excluded from the analysis, so spike autocorrelation was derived from a total of $n=6$ rats in dataset 1 . The decay of autocorrelation was fit by an exponential decay to the population of neurons; that is, the mean across autocorrelation functions from all units, for each time point and rat as follows:

$$
R(k \Delta)=A\left(e^{-\lambda k \Delta}+B\right),
$$

where $\lambda$ is the decay rate. Fitting was done using the Python (Python Software Foundation, version 2.7) function scipy.optimize.curve fit. As a minimal goodness of fit, we required $R^{2} \geq 0.5$, which excluded some rare outliers due to poor fits.

Recovery from large-activity events. MUA activity increased with negative LFP (nLFP) excursions (see Fig. 3). To analyze how MUA and LFP activity recovered from such large-activity events in dataset 1 , we identified these events of increased MUA activity in each channel and for each $4 \mathrm{~s}$ interval as nLFP excursions $\leqslant-2$ SDs after $z$-transformation (see Fig. $3 a)$. For each such nLFP event, both the LFP and the MUA in this channel for the time $\pm 100 \mathrm{~ms}$ around the most negative LFP excursion was then saved for further analysis. We required the next consecutive nLFP event to be at least $200 \mathrm{~ms}$ away. Next, the recovery from these nLFP was analyzed. nLFP segments were averaged for each time point (hour during sleep deprivation or after recovery period) and animal (see Fig. $3 b$ ) and fit by an exponential decay to derive recovery rates for each time point and each animal. The recovery rates $\lambda$ were derived by fitting an exponential function to the nLFP as follows:

$$
S(t)=A\left(e^{-\lambda t}+B\right)
$$

using the Python function scipy.optimize.curve fit. The recovery was fit from $30 \mathrm{~ms}$ after the most-negative LFP deflection to the local maximum within $100 \mathrm{~ms}$ after the most-negative LFP deflection because LFP sometimes exhibited a bleed-through of the spike trace in the vicinity of the most negative excursion (Ray et al., 2008; Nauhaus et al., 2009; Ray and Maunsell, 2011; Nauhaus et al., 2012). To avoid distortion by this distinct wave, we chose to fit the LFP recovery from large-activity events at $30 \mathrm{~ms}$ after the most-negative deflection.

Detection of bimodality in 2-6 Hz half-waves. For this analysis, LFP signals were further band-pass filtered $(2-6 \mathrm{~Hz})$ using a phase neutral filter by applying a second-order Butterworth filter in both directions in each $4 \mathrm{~s}$ interval. The histogram of positive $2-6 \mathrm{~Hz}$ half-waves exhibited a clear bimodality in double-logarithmic plot when the occasional brief large-amplitude activity events were not removed (dataset1; see above; Fig. 2c).

In dataset 1 , to test the dependence of our results on the occurrence of these events, all analyses were repeated for segments with or without this kind of large-amplitude slow-wave activity. Specifically, we determined whether the maximum of the $2-6 \mathrm{~Hz}$ filtered LFP signal in each channel and $4 \mathrm{~s}$ segment was larger than a given threshold and then separately analyzed spike autocorrelation and nLFP recovery for units and LFP data based on whether there was an event larger than this threshold. We set the threshold individually for each channel and each $4 \mathrm{~s}$ segment as the median plus $1 \mathrm{SD}$ of the positive $2-6 \mathrm{~Hz}$ half-waves (see Fig. $2 c$, vertical magenta line). This threshold reliably removed all large-amplitude events (i.e., the bimodality in the LFP amplitude distribution) while maintaining a large enough number of data segments for analysis both above and below threshold.

In dataset 2, segments during $\mathrm{Q}$ were carefully chosen not to contain any of these high-amplitude LFP events. This is evidenced by the $2-6 \mathrm{~Hz}$ LFP amplitude distribution, which did not exhibit a prominent bimodality (see Fig. $2 g$ ).

Computational model. To develop better insights into the determinants of timescale changes in cortical network activity, we studied a neuron network model that could exhibit long timescales associated with long-range temporal correlations. The neuron network model consisted of $N=100$ all-to-all coupled, binary-state neurons with the following dynamic rules: If neuron $j$ spiked at time $t$ (i.e., $s_{j}(t)=1$ ) as part of an active set of neurons $J(t)$, then a postsynaptic neuron $i$ will spike at time $t+1$ according to the following update rule:

$$
S_{i}(t+1)=\Theta\left[1-\prod_{j \in J(t)}\left(1-p_{i j}\right)-\zeta(t)\right]
$$

where $\Theta[x]$ is the unit step function and $\zeta(t)$ is a random number from a uniform distribution on $[0,1]$ to account for the probabilistic nature and variability of unitary synaptic efficacy. $p_{i j}$ is the asymmetric synaptic coupling strengths between each pair of neurons that are first drawn from a uniform distribution on $[0,1]$ and then multiplied by $N \cdot K / \sum p_{i j}$. The parameter $K$ is thus related to the average connectivity and is therefore a control parameter for the dynamics of the network: at $K=1$ each spiking neuron excites, on average, exactly one postsynaptic neuron meaning the network is critical. Conversely, at $K<1$, activity dies out prematurely and the system is subcritical. At $K>1$, each neuron excites, on average, more than on postsynaptic neuron and the system is supercritical (Shew et al., 2009). We instantiated the model at different average connectivities $K=1$ to study the spike autocorrelation and recovery from large-activity events in similar ways as in the experimental data. Network activity was introduced by randomly setting one neuron to active at each time step.

The temporal autocorrelation of neurons was studied by simulating network activity for 5050 time steps. After the initial 50 time steps, during which network activity typically reached a stable level, neuron activity was binned in 50-time-step bins, resulting in a spike count time series of length 100 for each neuron. This process was repeated for 100 network 


\section{Frontal}

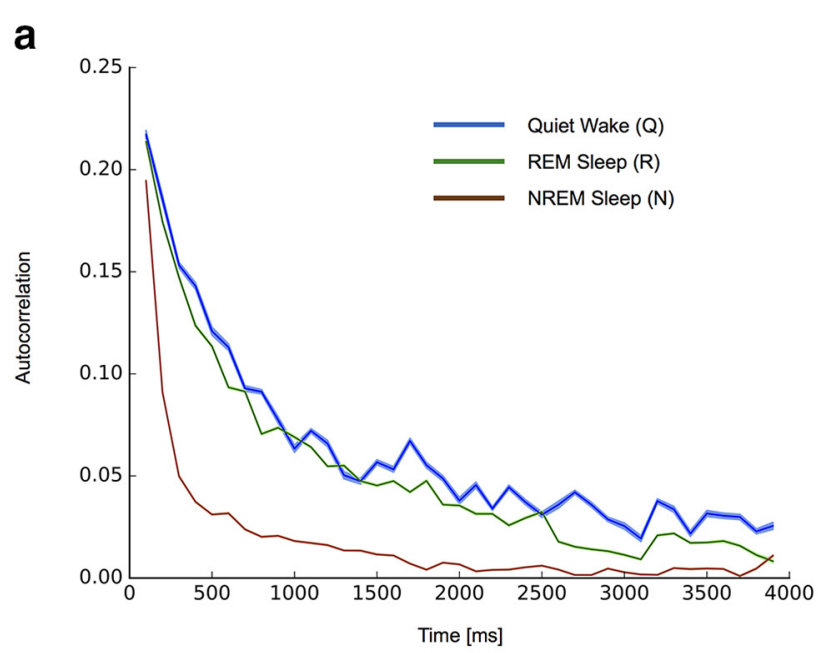

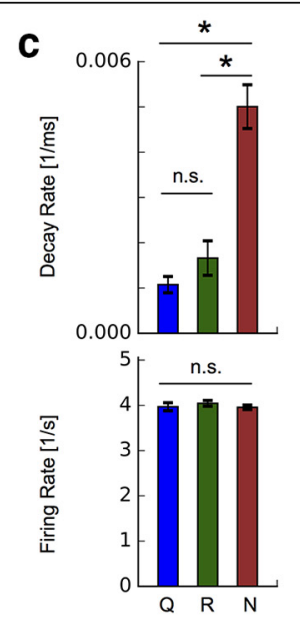

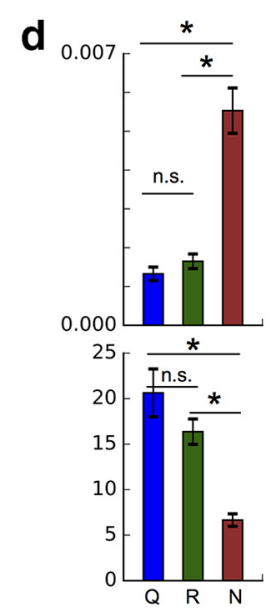

\section{Parietal}
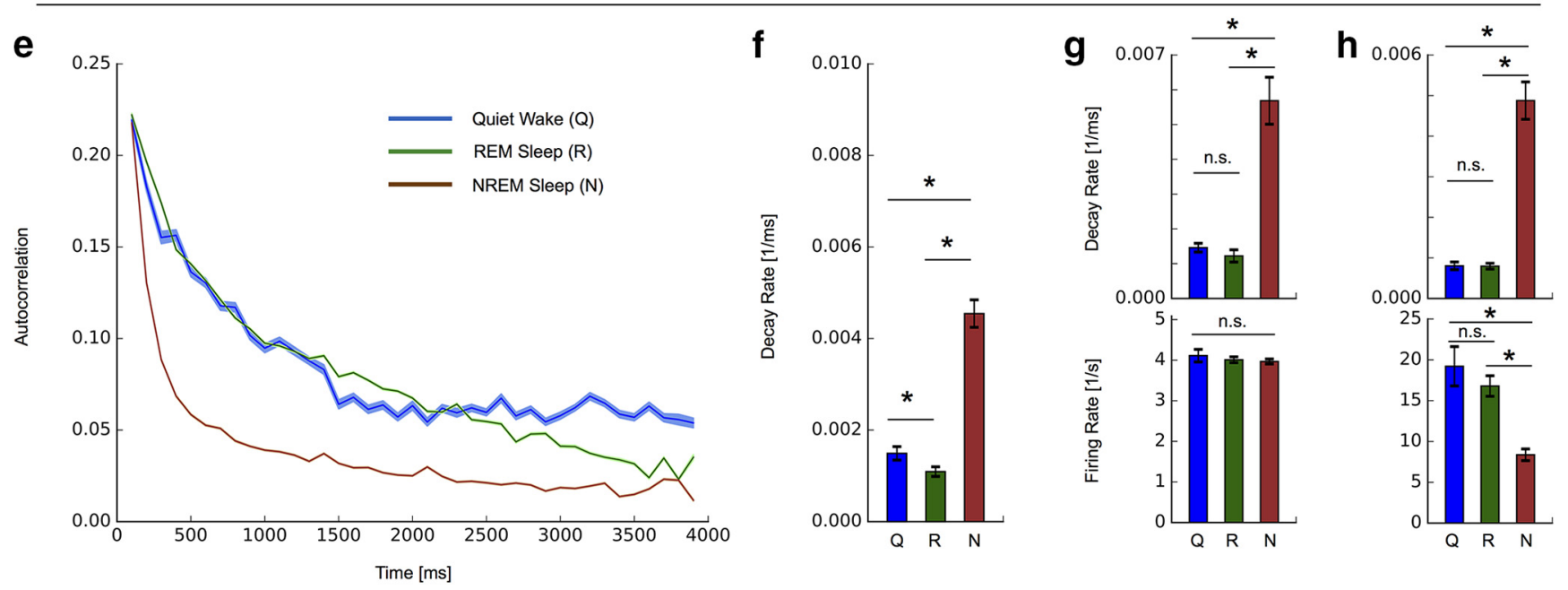

Figure 1. Breakdown of long-range temporal spiking correlations during NREM sleep in frontal and parietal cortex. $\boldsymbol{a}$, In frontal cortex, spike count autocorrelation (AC) decays rapidly in NREM sleep, whereas other vigilance states have similar slow decays. Mean AC over animals $(n=13 ; S E M ; S U A ;$ frontal cortex; $Q, R, N)$. $\boldsymbol{b}$, Summary of decay rates obtained from exponential fits over the whole autocorrelation function. $\boldsymbol{c}, \boldsymbol{d}$, Changes in autocorrelation decays observed in neurons maintaining a similar firing rate across vigilance states (c) and in neurons exhibiting a strong change in firing rate from wake to NREM sleep $(\boldsymbol{d})$. $\boldsymbol{e}-\boldsymbol{h}$, Breakdown of long-range temporal spiking correlation in NREM sleep in parietal cortex. Equivalent analysis as in $\boldsymbol{a}-\boldsymbol{d}$. All analyzed data are from dataset 2. $\star$ indicates a significance value $p<0.05$.

simulation runs, which allowed the calculation of Pearson's correlation coefficients $R$ (Eq. 1) for different time bins and across simulation runs analogously to the experimental data. Also analogously to experimental data, the population autocorrelation function was derived by averaging across all neurons and consequently fit by an exponential decay (Eq. 2) to derive a recovery rate $\lambda$.

To study the recovery from large-activity network events relevant to our experimental analysis, the recovery from large, intrinsic fluctuations in network activity was monitored. We simulated network activity for 5050 time steps. Network activity typically reached a stable level after $\sim 50$ time steps. From the following 5000 time steps, we identified large intrinsic fluctuations in the spike histogram greater than a threshold $T$, which we set to $T=2 \mathrm{SD}$. Once a large fluctuation of network activity in the spike histogram had been identified, we required the following fluctuation event to be at least 50 time steps apart. We averaged over all fluctuation events from a total of 100 network simulation. We fit an exponential decay (Eq. 3 ) to these averages to derive a recovery rate $\lambda$.

We implemented offline periods of spiking similar to those observed during sleep deprivation in two ways: (1) in an asynchronous manner in which the activity of an individual neuron was set to zero with a proba- bility $p_{\text {OFflne }}$ at each time step during the simulation and (2) in a synchronous manner in which all neurons at a time step were set to zero with a probability $p_{\text {OFFLINE }}$.

\section{Results}

We first assessed the timescales governing cortical dynamics at the individual neuron level in three different vigilance states: quiet wake, REM sleep, or NREM sleep. For each vigilance state, the spike count autocorrelation was calculated as the correlation coefficient between the number of putative single-unit spikes in pairs of time bins across all artifact-free $4 \mathrm{~s}$ segments. Following previous work (Ogawa and Komatsu, 2010; Murray et al., 2014), we derived an autocorrelation function reflective of the population- or network-level activity in each animal by averaging functions across units (Fig. 1a). We observed slow autocorrelation decays indicative of long-range temporal correlations during quiet wake in frontal cortex. Although REM sleep exhibited a similar, slowly decaying autocorrelation function, NREM sleep 
differed profoundly, exhibiting a fast decaying autocorrelation function at significantly higher rate (Fig. $1 b$; ANOVA: $F=87.2$, $p=1 \mathrm{e}-27$; post hoc unpaired $t$ test: $\mathrm{Q}$ vs $\mathrm{R} p=0.073$, Q vs $\mathrm{N} p=$ $7 \mathrm{e}-7, \mathrm{R}$ vs $\mathrm{N} p=3 \mathrm{e}-25)$. To ensure that these timescale differences were independent of firing rates, we repeated the analyses using only neurons with average firing rates between 3 and $5 \mathrm{~Hz}$ in all vigilance states. Although this selection abolished all firing rate differences between vigilance states, the autocorrelation decay differences were maintained (Fig. $1 c$; ANOVA: $F=14.8, p=$ $3 \mathrm{e}-6$; post hoc unpaired $t$ test: Q vs $\mathrm{R} p=0.42$, Q vs N $p=0.003$, $\mathrm{R}$ vs $\mathrm{N} p=1 \mathrm{e}-5)$. In order to assess whether timescale changes were also present in neurons undergoing strong firing rate changes between wake and NREM, we repeated our analysis on neurons that decreased firing by a factor of two or more from wake to NREM. Again, we observed that the faster autocorrelation decay in NREM compared with either wake or REM was maintained in these neurons exhibiting strong firing rate changes between vigilance states (Fig. $1 d$; firing rates - ANOVA: $F=30.0$, $p=6 \mathrm{e}-12$; post hoc unpaired $t$ test: $\mathrm{Q}$ vs $\mathrm{R} p=0.13$, Q vs $\mathrm{N} p=$ $5 \mathrm{e}-11$, $\mathrm{R}$ vs $\mathrm{N} p=4 \mathrm{e}-10$; autocorrelation decay rates - ANOVA: $F=22.6, p=2 \mathrm{e}-9$; post hoc unpaired $t$ test: $\mathrm{Q}$ vs $\mathrm{R} p=0.31$, Q vs $\mathrm{N} p=0.0001, \mathrm{R}$ vs $\mathrm{N} p=1 \mathrm{e}-7)$.

Next, we extended our analysis to recordings from parietal cortex to investigate how universal the vigilance-dependent timescale differences were across cortex. No statistically significant difference between frontal and parietal areas was observed when all quiet wake segments were compared (frontal: $0.0017 \pm 0.0002$ $1 / \mathrm{ms}$; parietal: $0.0015 \pm 0.00021 / \mathrm{ms} ; p=0.392$; unpaired $t$ test). In terms of timescale duration, the inverse of the autocorrelation decay rate, these observed rates correspond to values of several hundreds of milliseconds, which is in the order of magnitude also reported in areas of higher cortical hierarchy in nonhuman primates (Murray et al., 2014). Similar to frontal cortex, we observed a much more rapid spike count autocorrelation decay during NREM sleep compared with quiet wake and REM sleep, indicative of a loss of long-range temporal correlations (Fig. 1e,f; ANOVA: $F=51.5, p=1 \mathrm{e}-17$; post hoc unpaired $t$ test: $\mathrm{Q}$ vs $\mathrm{R} p=$ $0.042, \mathrm{Q}$ vs $\mathrm{N} p=5 \mathrm{e}-4, \mathrm{R}$ vs $\mathrm{N} p=7 \mathrm{e}-17)$. Again, this difference was maintained when only select neurons with similar firing rates were considered (Fig. 1g; ANOVA: $F=15.3, p=6 \mathrm{e}-6$; post hoc unpaired $t$ test: $\mathrm{Q}$ vs $\mathrm{R} p=0.633, \mathrm{Q}$ vs $\mathrm{N} p=0.039$, $\mathrm{R}$ vs $\mathrm{N} p=$ $3 e-6)$. In further agreement with frontal cortex, a faster autocorrelation decay in NREM compared with either wake or REM was maintained when only neurons with a drastic firing rate change between vigilance states were considered (Fig. $1 \mathrm{~h}$; firing rates ANOVA: $F=22.5, p=3 \mathrm{e}-9$; post hoc unpaired $t$ test: $\mathrm{Q}$ vs $\mathrm{R} p=$ $0.36, \mathrm{Q}$ vs $\mathrm{N} p=9 \mathrm{e}-8, \mathrm{R}$ vs $\mathrm{N} p=1 \mathrm{e}-8$; autocorrelation decay rates $-F=41.3, p=4 \mathrm{e}-15$; post hoc unpaired $t$ test: $\mathrm{Q}$ vs $\mathrm{R} p=$ $0.94, \mathrm{Q}$ vs $\mathrm{N} p=1 \mathrm{e}-5, \mathrm{R}$ vs $\mathrm{N} p=9 \mathrm{e}-13$ ).

These results indicate distinct timescales in different vigilance states: long timescales associated with a slow autocorrelation decay during quiet wake and REM sleep and short timescales during NREM sleep. We next investigated how persistent these timescales were within each vigilance state across time. Extended wakefulness, for example, might affect the capacity of brain networks to integrate and process information effectively as measured by cortical timescales. Previous work has shown that wake is not a homogeneous state. Spontaneous waking is associated with more pronounced slow rhythms in the EEG (Finelli et al., 2000; Strijkstra et al., 2003), changes in firing rates (Fisher et al., 2016), and the occurrence of local OFF periods when neurons stop firing and go "offline" (Poulet and Petersen, 2008; Vyazovskiy et al., 2011; McGinley et al., 2015). Some of these changes, like local offline periods, may not show in scalp EEG (Vyazovskiy et al., 2011); the underlying state may thus still be considered wake in the traditional sense. We therefore studied neuron timescale dynamics in wake by systematically taking the effect of offline periods into consideration.

We first analyzed MUA of frontal cortex in freely behaving rats during $6 \mathrm{~h}$ of sleep deprivation and after a consecutive recovery period (dataset 1 ). In these data, the spike autocorrelation functions exhibited a progressively faster decay during sleep deprivation (Fig. 2a). Statistical analysis revealed the autocorrelation function decay rates to increase significantly during sleep deprivation and to return to a slower rate after consecutive recovery sleep (Fig. $2 b$; ANOVA: $F=2.91, p=0.015$; post hoc twotailed paired $t$ test: SD0 vs SD6 $p=0.002$, SD6 vs REC $p=0.01$ ). Similar to previous work (Vyazovskiy et al., 2011), we observed occasional large positive LFP half-waves in the $2-6 \mathrm{~Hz}$ frequency band that were accompanied by pauses in the MUA (Fig. 2c). When plotted in double logarithmic coordinates, the amplitudes of positive half-waves in this frequency band exhibited a distinct bimodality in their density distribution (Fig. 2c). Such a bimodality has also been observed in other frequency bands, where it was taken as indication for the existence of two coexisting different states between which dynamics can switch (Freyer et al., 2011). In our case, the observed bimodality may similarly suggest two distinct states characterized by different low-frequency power levels. We hypothesized that large-amplitude LFP events might correspond to a more sleep-like state and thus potentially imprint shorter timescales. We thus repeated our analysis in select data with or without any such events. Specifically, we defined a threshold for each animal and channel individually that separated the two peaks in the LFP amplitude distribution (Fig. $2 c$, right, magenta line). We then analyzed spike count autocorrelations separately in all channel segments in which amplitudes in the $2-6 \mathrm{~Hz}$ frequency band either exceeded this threshold at least once (number of 4 s channel segments; SD0-SD6, REC; mean \pm SEM; $5329 \pm 2868,4977 \pm 2402,4515 \pm 1708,3948 \pm 1231$, $3692 \pm 1656,2369 \pm 443,2454 \pm 479,3009 \pm 874)$ or stayed below it throughout the whole $4 \mathrm{~s}$ segment (number of $4 \mathrm{~s}$ channel segments; SD0-SD6, REC; mean \pm SEM; $734 \pm 491,646 \pm$ $363,694 \pm 321,656 \pm 247,560 \pm 308,399 \pm 91,362 \pm 98,350 \pm$ $75)$. The separation of sleep deprivation data along these two peaks in the LFP distribution revealed that timescale changes were only apparent in segments that contained high-amplitude LFP events (Fig. 2e; ANOVA: $F=3.4, p=0.006$; post hoc twotailed paired $t$ test: SD0 vs SD6 $p=0.002$, SD6 vs REC $p=0.003$ ). Timescales did not change during sleep deprivation in the lowamplitude regime (Fig. $2 d$; ANOVA: $F=0.47, p=0.853$ ).

Based on these observations, we hypothesized that the intermittent large-amplitude LFP events associated with paused spiking might cause a decrease in temporal correlations between adjacent data segments and thereby lead to the observed faster spike count autocorrelation decays. To test this hypothesis, we calculated spike count correlations between time bins (time bin 1 and time bin 2; width 100 ms each; containing no large-amplitude LFP events; Fig. $2 f$ ) that were intervened by an interval of $200 \mathrm{~ms}$ (with or without large-amplitude LFP events) during the last hour of sleep deprivation (SD6). We then compared the spike count correlations between the two time bins depending on whether the intervening $200 \mathrm{~ms}$ interval contained one or more large-amplitude LFP events. Spike count correlation values were significantly lower when the intervening interval contained large-amplitude LFP events (Fig. $2 f ; p=0.021$; two-tailed paired $t$ test). Together, these observations indicate distinct dynamics with respect to the two 

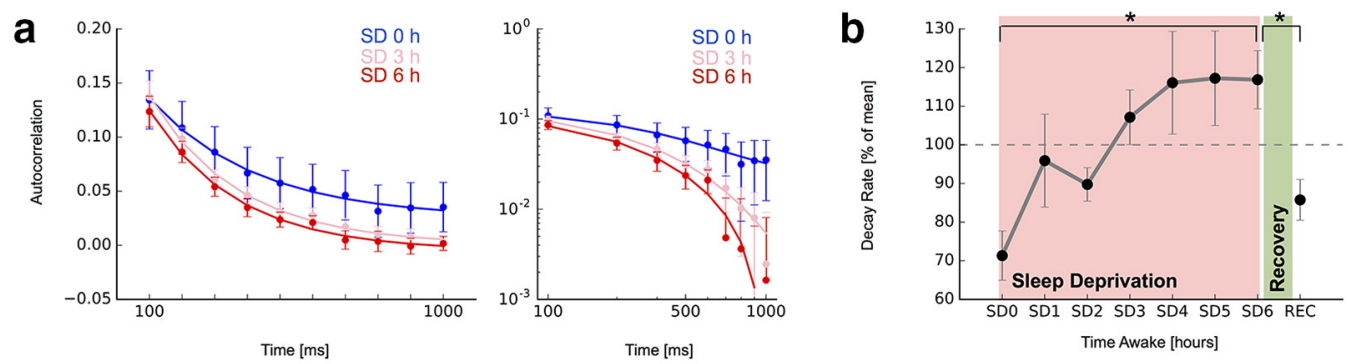

\section{C}

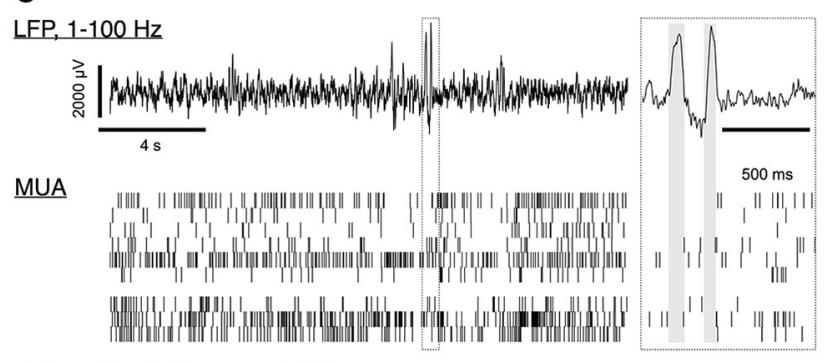

LFP positive half-waves, $2-6 \mathrm{~Hz}$
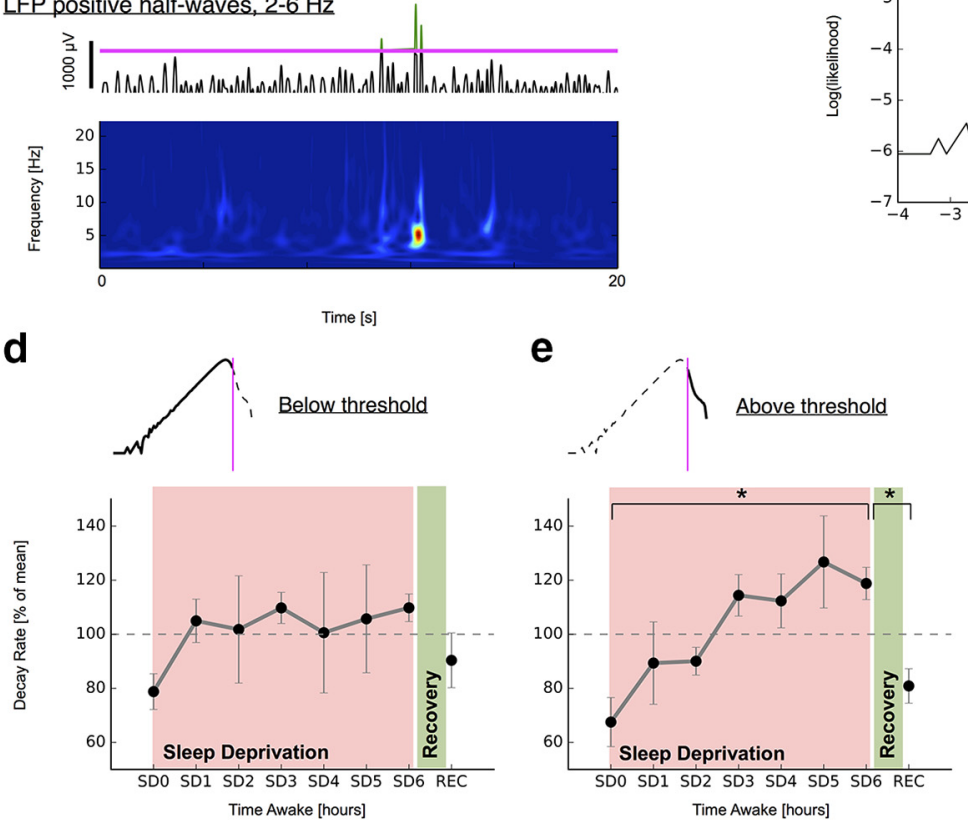

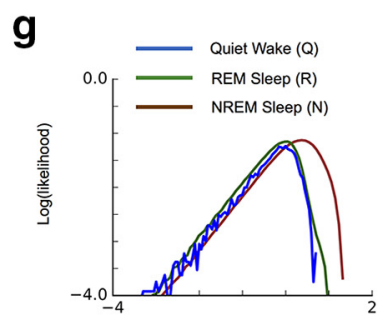

Log(amplitude)
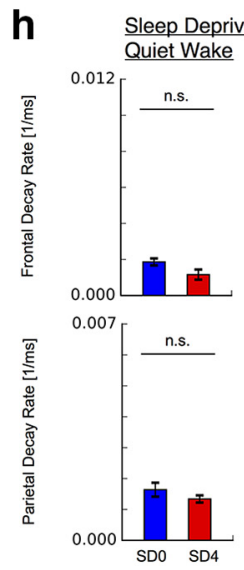
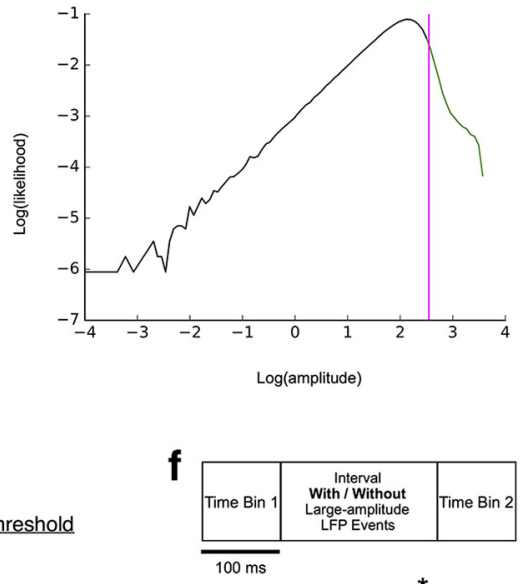

Log(amplitude)

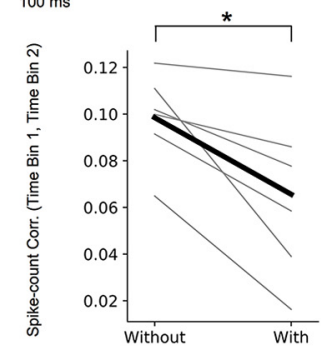

NREM Sleep

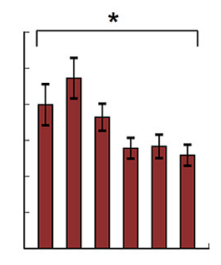

illiting

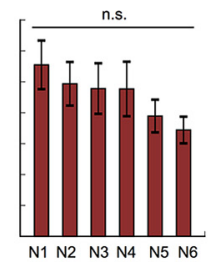

Figure 2. Two-state dynamics underlies the decline in long-range temporal correlations during sleep deprivation. $\boldsymbol{a}$, Decay in spike count autocorrelation (AC) accelerates as a function of time awake and recovers after sleep (MUA; $Q ; S D 0-S D 6$ followed by REC). Mean AC over all multiunits ( $n=26 ; 1$ rat; SEM). Solid lines indicate exponential fit. Left, Linear plot. Right, Double-logarithmic plot. SD3 line has been shifted slightly upward for visibility. $\boldsymbol{b}$, Summary of decline in decay rates and recovery for all $n=6$ animals (SEM). $\boldsymbol{c}$, Identification of two-state dynamics underlying decay in long-range temporal correlations with sleep deprivation. Large-amplitude LFP events are observed intermittently and typically coincide with pauses in MUA (top; offline periods marked as gray areas in magnified inset). These LFP events typically present as one or more positive half-waves predominantly in the frequency range $2-6 \mathrm{~Hz}$ (bottom, wavelet (Figure legend continues.) 
peaks in the LFP distribution and identify the intermittent largeamplitude LFP events (i.e., offline periods) as a cause for the decreased temporal correlations between successive data segments and, consequently, the faster autocorrelation decay.

To further confirm the causal role of intermittent largeamplitude LFP events for the apparent timescale decline during extended wake, we repeated our analysis in dataset 2 , in which such large-amplitude LFP events had been excluded altogether in the selection of quiet wake data (as confirmed by the absence of a distinct bimodality in the LFP amplitude distribution; Fig. $2 g$, blue). Therefore, autocorrelation decays obtained from quiet wake exhibited no statistically significant change during sleep deprivation in this dataset (frontal and parietal; SD0 vs SD4; unpaired $t$ test; Fig. $2 h$ ). During extended wake, the occurrence of shorter timescales is thus linked to the presence of intermittent large-amplitude LFP events. Over the course of NREM sleep, autocorrelation decays became significantly less steep in frontal cortex (Fig. $2 j$; ANOVA: $F=3.84, p=0.003$; post hoc unpaired $t$ test: N1 vs N6 $p=0.039$ ). A similar trend was visible in parietal cortex, although it did not reach the significance level there. REM sleep, conversely, did not exhibit a significant change in any region (Fig. $2 i$ ). The differential changes observed over the course of NREM sleep, but not REM sleep, thus provide further evidence for the relevance of low-frequency large-amplitude events as the essential mechanism for the apparent timescale change. Lowfrequency large-amplitude LFP events were present during NREM but not REM sleep (Fig. $2 g$, brown vs green line). The well known decline of low-frequency activity over the course of NREM sleep (Borbély et al., 1981; Finelli et al., 2000; Vyazovskiy et al., 2011) can thus account for the observed gradual restoration of slow autocorrelation decays. Prolonged waking is also known to induce an increase in slow-wave activity most prominently over frontal cortex areas (Werth et al., 1997; Finelli et al., 2001) and can thus account for the more pronounced reduction in decay rates in frontal compared with parietal cortex during recovery sleep (Fig. 2j).

There exists an intimate relationship between the autocorrelation function governing spontaneous fluctuations and the time that it takes a signal to recover from small perturbations (Wissel, 1984; Ives, 1995; Scheffer et al., 2009). From this dynamical systems perspective, a slow autocorrelation decay also implies a slow recovery from perturbations or intrinsic fluctuations. We hypothesized that equivalent wake-time dependent changes quantified by autocorrelation decays should also manifest in recovery rates from intrinsic fluctuations. We first identified sizeable negative excursions from the mean in each LFP channel (Fig. 3a). Next, we averaged across all these LFP segments for each rat and at each time point (dataset 1) to quantify the return to baseline activity during sleep deprivation and after consecutive recovery sleep. Negative LFP averages typically coincided with increased

$\leftarrow$

(Figure legend continued.) spectrogram and positive LFP trace band-pass filtered between 2 and $6 \mathrm{~Hz}$ ). Right, Bimodality in the histogram of positive $2-6 \mathrm{~Hz}$ waves. The bimodality was used to separate the large-amplitude LFPs containing putative offline periods from other activity for each rat individually (magenta line). $\boldsymbol{d}, \boldsymbol{e}$, Decline in long-range temporal correlations is restricted to data segments containing large-amplitude LFP events. Data depicted in $\boldsymbol{a}-\boldsymbol{d}$ are from dataset $1 . f$, Intermittent large-amplitude LFP events decrease spike count autocorrelation (gray: individual animals; black: mean across animals). $\boldsymbol{g}$, Histogram of positive LFP 2-6 Hz waves sorted by vigilance states (dataset 2; top row, frontal; bottom row, parietal). $\boldsymbol{h}$, Autocorrelation decays remains unchanged (right) in data without large-amplitude LFP events during sleep deprivation ( $Q$, SDO, SD4). $\boldsymbol{i}$, Autocorrelation decay rates during REM sleep remain unchanged (dataset 2; 6 h recovery sleep; R1-R6).j, Autocorrelation decay rates in NREM sleep decrease over the $6 \mathrm{~h}$ recovery sleep (dataset $2 ; \mathrm{N1}-\mathrm{N} 6$ ). $\star$ indicates a significance value $p<0.05$. spiking activity $($ Fig. $3 b, c)$. To quantify the recovery rate by which LFPs returned to baseline after a negative excursion, we fit the return with an exponential function. Recovery rates obtained from these fits increased during sleep deprivation and reversed to lower values after consecutive sleep (Fig. 3d,e; ANOVA, $F=3.5$, $p=0.004)$. Post hoc statistical comparison between the beginning (SD0) and the end of sleep deprivation (SD6) and between the end of sleep deprivation (SD6) and after recovery sleep (REC) revealed both changes to be significant ( $p=0.002$ and $p=0.01$, respectively; two-tailed paired $t$ test). We hypothesized that largeamplitude LFP events would again drive the observed timescale decline. As with spike count autocorrelation, we thus repeated our analysis for both the low-amplitude and the high-amplitude LFP regimes separately (number of detected nLFP events per $4 \mathrm{~s}$ segment and channel; SD0-SD6, REC; mean \pm SEM; segments without high-amplitude LFP: $4.39 \pm 0.10,4.25 \pm 0.18,4.34 \pm 0.15$, $4.44 \pm 0.13,4.47 \pm 0.13,4.40 \pm 0.15,4.44 \pm 0.14,4.36 \pm 0.12$ segments with high-amplitude LFP: $3.54 \pm 0.18,3.36 \pm 0.23,3.37 \pm$ $0.19,3.47 \pm 0.18,3.51 \pm 0.16,3.40 \pm 0.16,3.50 \pm 0.17,3.29 \pm 0.18$ ). Note that nLFP events are defined relative to an amplitudedependent threshold in each $4 \mathrm{~s}$ segment individually to allow comparison across conditions. In agreement with our observations from spike count autocorrelations, timescale changes were only apparent in segments also containing high-amplitude LFP events (ANOVA: $F=2.79, p=0.02$; post hoc two-tailed paired $t$ test: $\mathrm{SD} 0$ vs $\mathrm{SD} 6 p=0.02$, SD6 vs REC $p=0.02$ ), whereas timescales did not change significantly during sleep deprivation in the low-amplitude regime (Fig. 3f,g; ANOVA: $F=0.54, p=0.797$ ).

Together, these results indicated that intermittent periods of large-amplitude LFP can lead to an apparent decline of long cortical timescales during wake. The concomittent recording of unit activity suggested that these LFP events are characterized by pauses in neuron spiking. To better understand how these intermittent offline periods could account for the observed timescale changes, we studied a neuron network model exhibiting long timescales associated with long-range temporal correlations analogous to experiment. Specifically, the network's average connectivity was set so that network dynamics was located right at the transition between an inactive and an active network phase (Shew et al., 2009). At this transition state, the network exhibited long timescales associated with both the autocorrelation decay and the recovery from intrinsic fluctuations that are purely based on reverberations in the network supported by recurrent connectivity. We then affected the ability of the network to sustain reverberations by allowing neurons to go offline and thereby not participate in reverberating activity. Specifically, we included the possibility that neurons could go offline either in a synchronous, probabilistic manner or, in another instantiation of the model, in an asynchronous, local manner, inspired by reports in Vyazovskiy et al. (2011). We hypothesized that these interruptions of reverberating activity through intermittent offline periods would be reflected in the network autocorrelation and recovery from intrinsic fluctuations, where they would lead to faster decays and recovery, respectively. Our simulations demonstrated that the ability of neurons to go offline, whether asynchronously or synchronously, was sufficient to reproduce our experimental findings. In particular, with higher probability for neurons to go offline, faster decays in spike count autocorrelation and a more rapid recovery from intrinsic fluctuations were observed as a result of the disruption of the reverberating activity in the network (Fig. 4). In combination with experimental results, these modeling results thus suggest that presence of the offline periods caused by an increasing tendency for neurons to go offline, whether locally 

LFP 2 SD

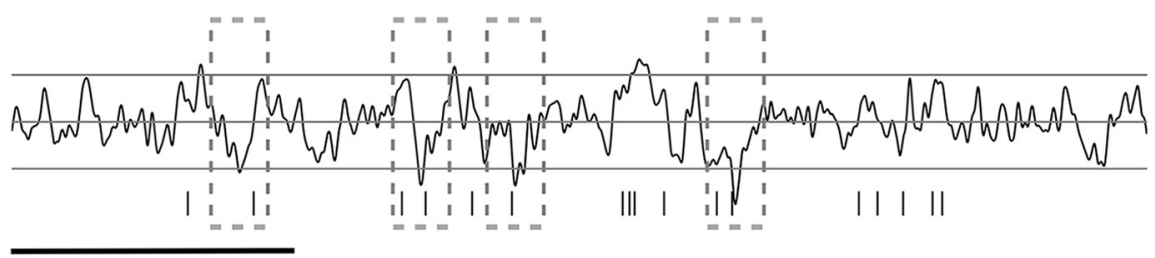

b

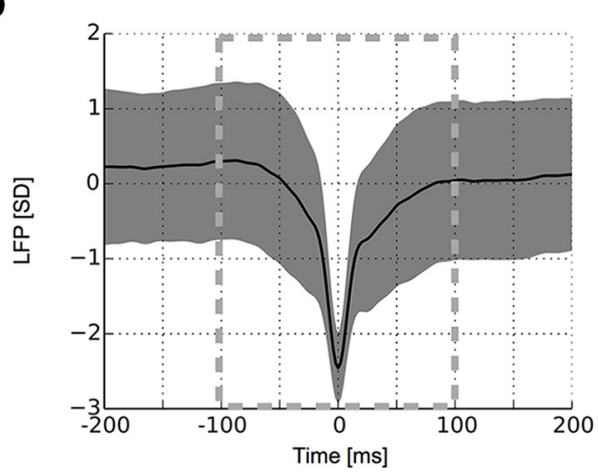

d

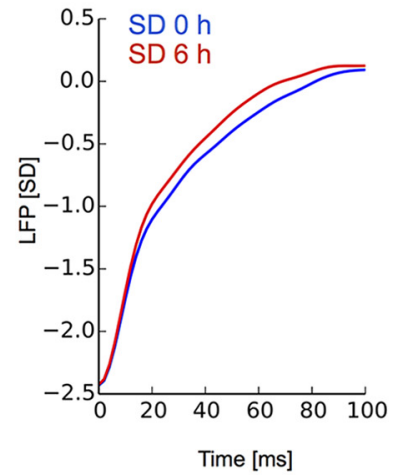

f

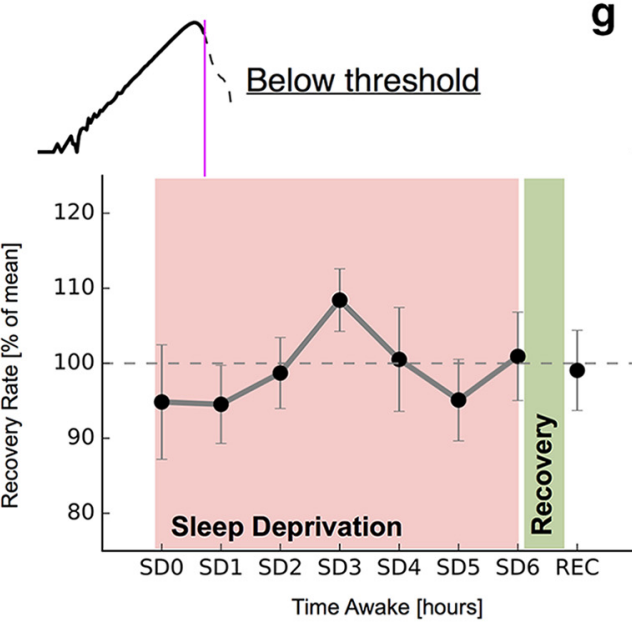

C

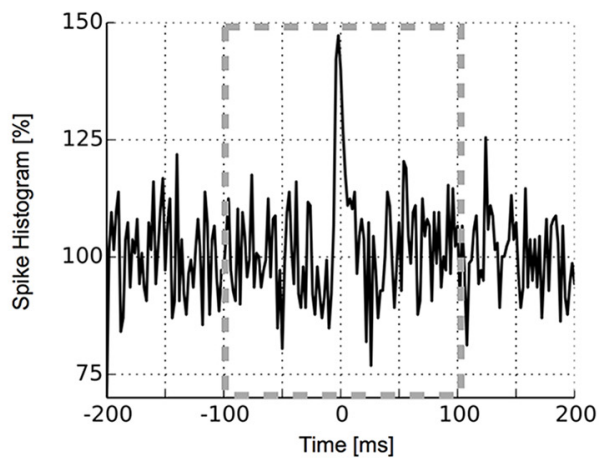

e

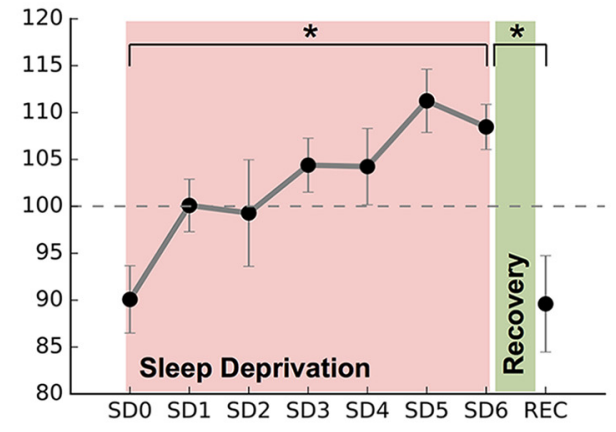

Time Awake [hours]

g

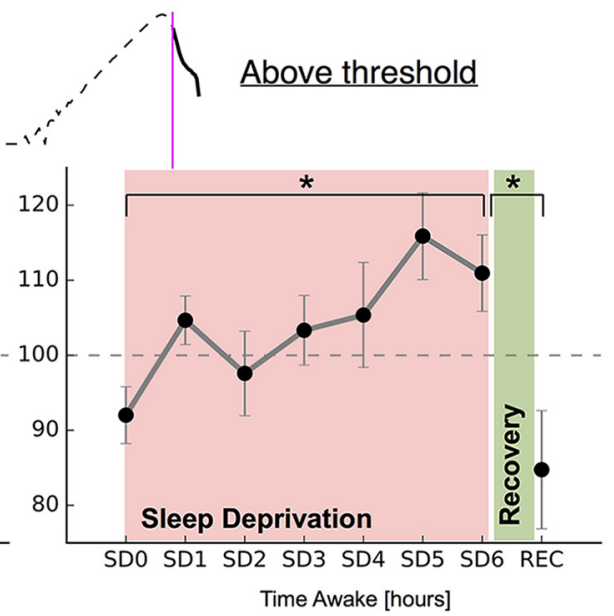

Figure 3. Two-state dynamics underlie the accelerated recovery from large-activity events during sleep deprivation. $\boldsymbol{a}$, Identification of large-activity events from LFP. nLFP ( $\leqslant 2$ SDs) deflections were averaged over each time point during the sleep deprivation experiment (SD0-SD6, REC) and for each rat. $\boldsymbol{b}$, Mean nLFP over all events and channels (1 rat; SEM). $\boldsymbol{c}$, Corresponding nLFP-triggered spike histogram. The histogram is normalized to mean activity from -150 to $-100 \mathrm{~ms}$. $\boldsymbol{d}$, Recovery from intrinsic nLFP excursions accelerates as a function of time awake and recovers after sleep. Mean $n L F P$ traces from the beginning and end of sleep deprivation from one rat are shown. $\boldsymbol{e}$, Summary of nLFP recovery rates for all $n=7$ animals (SEM). $\boldsymbol{f}, \boldsymbol{g}$, More rapid recovery from large-activity events is restricted to data segments containing large-amplitude LFP events. All analyzed data are from dataset 2 . $\star$ indicates a significance value $p<0.05$. 


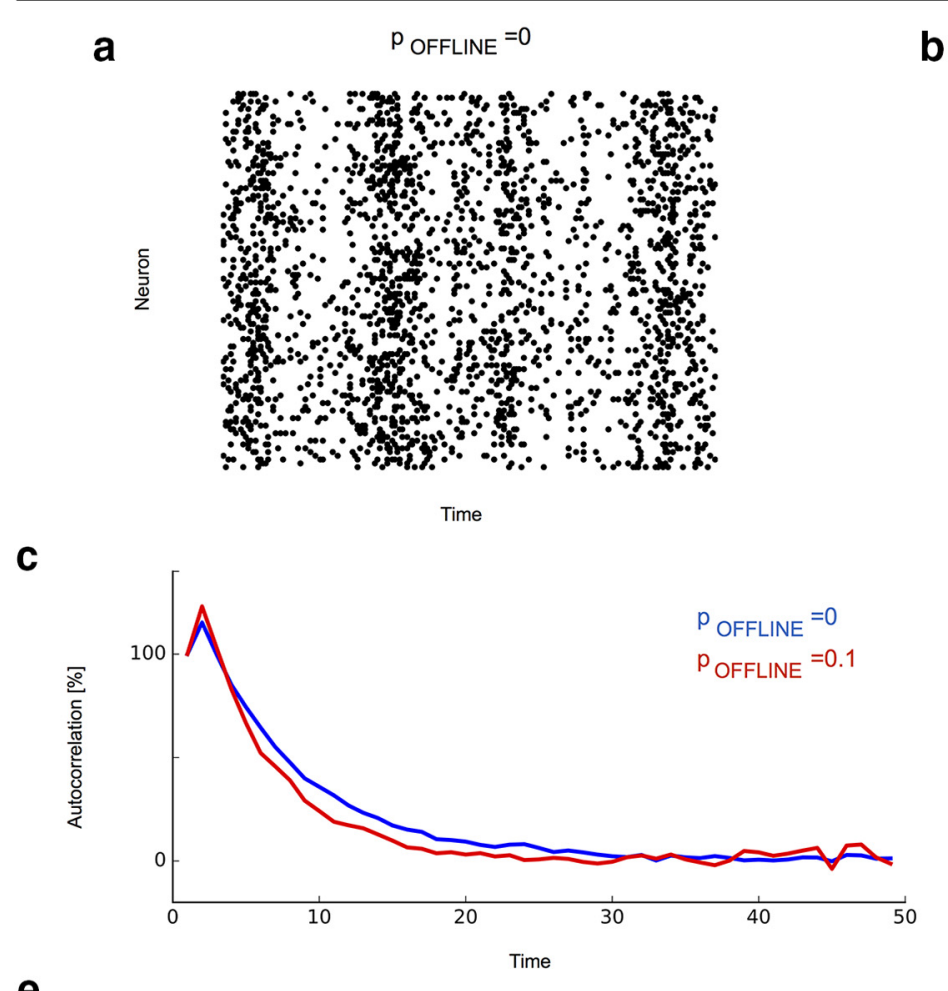

e

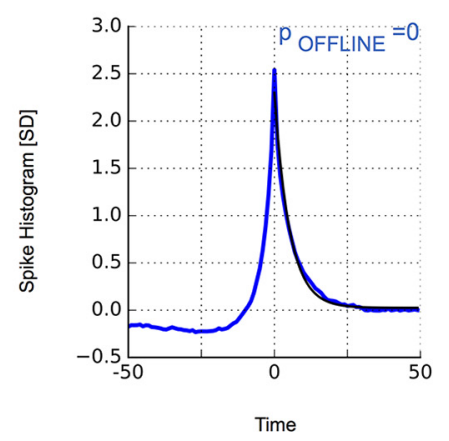

b

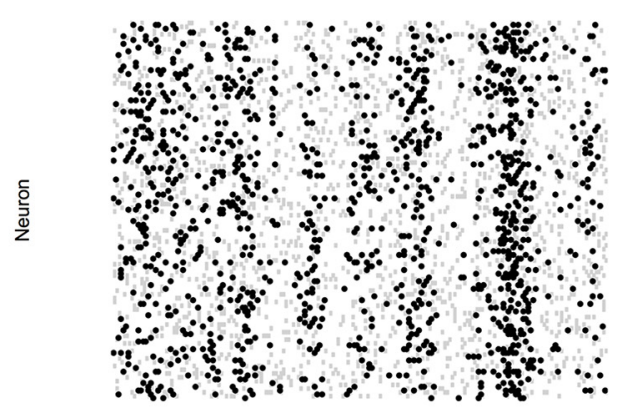

Time

d

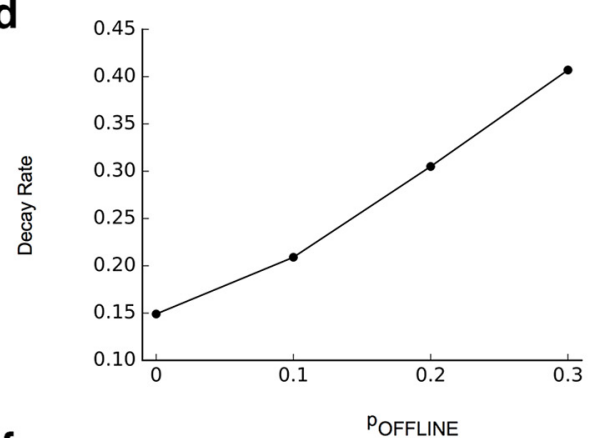

f

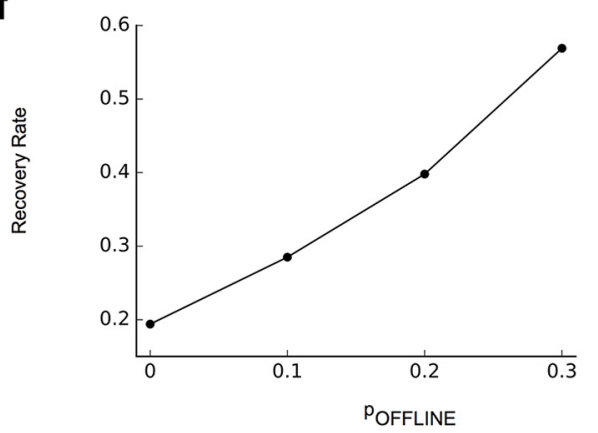

Synchronous OFFLINE Periods

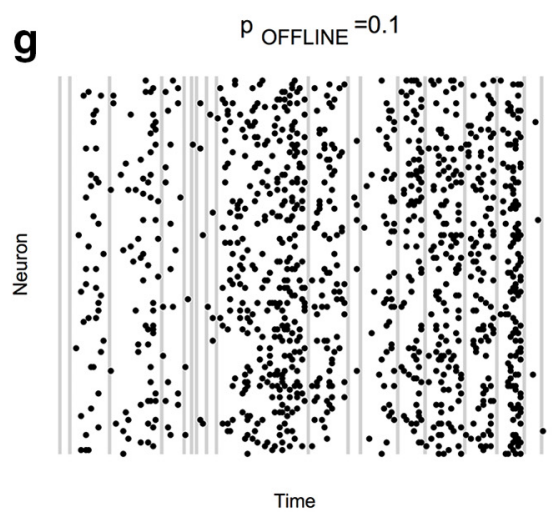

h

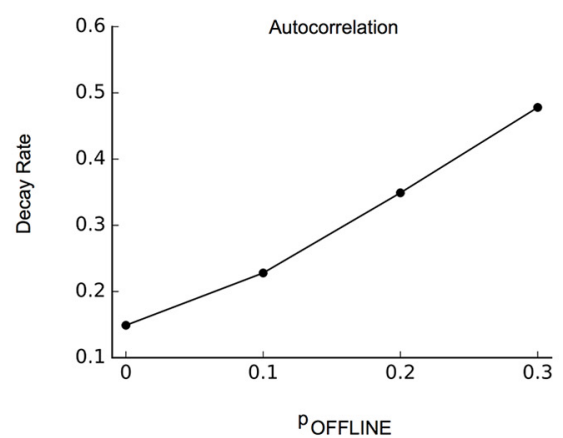

i

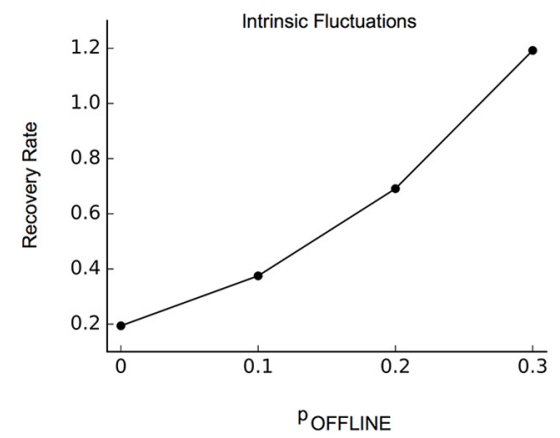

Figure 4. Neuron network model with the ability of neurons to go offline captures the differential decline of long-range temporal correlations. The network was instantiated at a connectivity $K=$ 1 and with different probablities $p_{\text {OFFLINE }}$ for neurons to go offline for a brief amount of time in an asynchronous $(\boldsymbol{a}-\boldsymbol{f})$ or synchronous $(\boldsymbol{g}-\boldsymbol{i})$ manner. $\boldsymbol{a}$, Raster plot without the presence of offline periods. $\boldsymbol{b}$, Raster plot with asynchronous offline periods ( $p_{\text {OFFLINE }}=0.1$, gray shaded areas). $\boldsymbol{c}$, Autocorrelation functions for spiking activity in a network without offline periods ( $p_{\text {OFFLINE }}=0$, blue) and in a network with asynchronous offline periods ( $p_{\text {OFFLnE }}=0.1$, red) in neuron activity. $\boldsymbol{d}$, Autocorrelation decay rates increase with higher probability for asynchronous offline periods. $\boldsymbol{e}$, Recovery from intrinsic fluctuations ( $p_{\text {OFFLINE }}=0$, blue; $p_{\text {OFFLINE }}=0.1$, red). $f$, Recovery rates from intrinsic fluctuations increase with higher probability for asynchrnous offline periods. $\boldsymbol{g}$, Raster plot with synchronous offline periods (gray shaded areas). $\boldsymbol{h}, \boldsymbol{i}$, Autocorrelation decay and recovery rates from intrinsic fluctuations increase with higher probability for synchronous offline periods. 
(e.g., during sleep deprivation) or in synchrony (e.g., during NREM sleep), is sufficient to account for the shortened timescales. This conclusion is based on our experimental observations, in which the timescale changes were only observed in data segments containing neuronal offline and where intermittent offline periods decreased temporal correlations. The causal role of offline periods is furthermore supported by modeling in which the intrusion of offline periods was sufficient to disrupt the long-range temporal correlations brought about by reverberating activity in the recurrent network.

\section{Discussion}

The ability to integrate information over time is a prerequisite for brain function during wake. Information integration is believed to be supported by long timescales associated with long-range temporal correlations in neuron-spiking activity. We here provide first-time evidence at the neuronal level that these timescales change as a function of vigilance state and time awake. Although quiet wake and REM sleep are characterized by similar, long timescales, the long timescales are abrogated during NREM sleep, which may explain the lack of responsiveness and loss of consciousness in this state. During wake and in particular during sleep deprivation, we observe that cortex dynamics can exhibit rapid, intermittent transitions between these different states; that is, states with long timescales interrupted by brief, sleep-like epochs governed by short timescales. During sleep deprivation, this interplay between states gives rise to an overall progressive decline of timescales with negative consequences for the network's capacity to effectively integrate and process information. At a mechanistic level, we identify the ability of neurons to go offline as a determining factor of the disruption of long-range temporal correlations established by reverberating network activity. Conclusively, our work provides novel mechanistic and functional links among behavioral manifestations of sleep, waking, and sleep deprivation and specific measurable changes in the network dynamics relevant for characterizing the brain's changing ability to integrate and process information over time and across vigilance states.

The present work provides a missing link between long cortical timescales during wake (Ogawa and Komatsu, 2010; Honey et al., 2012; Stephens et al., 2013; Murray et al., 2014; Chaudhuri et al., 2015) and reports of disrupted long-range correlations during NREM sleep (Massimini et al., 2005; Tagliazucchi et al., 2013). The observed breakdown of long timescales in ongoing spiking activity here provides insights that complement these reports, which were based on large-scale signals measured by EEG and fMRI. From a theory point of view, the timescales quantified by the autocorrelation of intrinsic network activity are intimately linked to the timescales that control the response to external perturbations in the same network (Wissel, 1984; Ives, 1995; Scheffer et al., 2009; Meisel et al., 2015). The fast autocorrelation decay observed in our data during NREM sleep thus links to observations of reduced stimulation responses using TMS in the same vigilance state (Massimini et al., 2005). Together, these findings suggest that NREM sleep is not compatible with integrating information and may explain the lack of responsiveness or loss of consciousness.

In detailed analyses of the apparent gradual timescale decline during extended wake, we identified intermittent transitions between two different states. We found that the state characterized by a fast decaying autocorrelation increasingly dominated the overall dynamics, whereas autocorrelation decay rates did not change for the low-amplitude state during sleep deprivation. The two peaks visible in the LFP amplitude distribution thus point to distinct dynamical states governed by long timescales in the lowamplitude regime and shorter timescales in the high-amplitude regime. The identification of two different states being at play relied on the ability to record data at high temporal and spatial resolution in our data. It is therefore possible that the gradual decline in timescales observed during the transition into NREM sleep (Tagliazucchi et al., 2013) or during human sleep deprivation (Meisel et al., 2017) is similarly the result of two different coexisting states, which more coarse methods such as fMRI or scalp EEG may not be able to resolve in the temporal domain. Several subcortical structures in the brainstem, hypothalamus, and basal forebrain are known to regulate the maintenance of waking and sleep states through neuromodulatory action (Jones, 2005). It is conceivable that these subcortical structures control the transient occurrences of the large-amplitude LFP epochs observed in some of our data during wake.

Functionally, the shorter timescales during NREM sleep indicate a severely reduced ability to integrate information. During sleep deprivation, shorter timescales induced by intermittent, sleep-like, high-amplitude LFP events (offline events at the neuronal level) may be similarly detrimental for cortical networks to process information. Our work links the decline of optimal brain functioning during sleep deprivation (Van Dongen et al., 2003; Banks and Dinges, 2007; Mignot, 2008; Killgore, 2010; Lo et al., 2012) to a growing body of research that has identified longrange temporal correlation in brain activity as an essential ingredient for cortical information processing. Furthermore, the observation that long-range temporal correlations are disrupted by intermittent states characterized by neuronal offline periods provides mechanistic insight, which can help to explain some performance observations during sleep deprivation. Although it is well established that performance as, for example, quantified in the psychomotor vigilance test (PVT), decreases, on average, as a function of time awake, a subject may still perform almost normally even after extended periods of sleep deprivation during individual trials in the PVT (Banks and Dinges, 2007). It is conceivable that normal performance is still feasible at times when timescales and information integration are not compromised by intermittent high-amplitude events, whereas performance will decrease on average due to the growing dominance of intermittent epochs that disrupt long timescales during sleep deprivation.

Long-range temporal correlations are generic features that arise when a system is in the vicinity of a critical state (Bak et al., 1987). Mounting evidence from computational and experimental studies indicates that cortical neural networks operate at some sort of critical state (Bornholdt and Rohlf, 2000; Beggs and Plenz, 2003; Haldeman and Beggs, 2005; Shew et al., 2009; Meisel et al., 2012; Markram et al., 2015). The observation of long network timescales during wake is in agreement with this criticality hypothesis (Linkenkaer-Hansen et al., 2001) and indicates that the ability to maintain memory of past activity is preserved in neural systems across different spatial scales (Meisel et al., 2015). Conversely, fast-decaying autocorrelation functions such as during NREM sleep and also during sleep deprivation, as reported here, may be seen as indication for a dynamical state further away from criticality or where critical dynamics are disrupted by some other process. In a previous study based on EEG, we reported fading signatures of critical dynamics during sleep deprivation (Meisel et al., 2013). Our results at the individual neuron level here complement these findings by demonstrating the increased disruption of long timescales (or critical dynamics) during sleep deprivation through intermittent offline periods. Within this 
framework, our observations support an intriguing hypothesis for a function of sleep: to reorganize cortical networks toward critical dynamics with long-range temporal correlations for optimal functioning and information processing during wake.

\section{References}

Bak P, Tang C, Wiesenfeld K (1987) Self-organized criticality: an explanation of the 1/f noise. Phys Rev Lett 59:381-384. CrossRef Medline

Banks S, Dinges DF (2007) Behavioral and physiological consequences of sleep restriction. J Clin Sleep Med 3:519-528. Medline

Beggs JM, Plenz D (2003) Neuronal avalanches in neocortical circuits. J Neurosci 23:11167-11177. Medline

Borbély AA, Baumann F, Brandeis D, Strauch I, Lehmann D (1981) Sleep deprivation: effect on sleep stages and EEG power density in man. Electroencephalogr Clin Neurophysiol 51:483-495. CrossRef Medline

Bornholdt S, Rohlf T (2000) Topological evolution of dynamical networks: Global criticality from local dynamics. Phys Rev Lett 84:6114-6117. CrossRef Medline

Chaudhuri R, Knoblauch K, Gariel MA, Kennedy H, Wang XJ (2015) A large-scale circuit mechanism for hierarchical dynamical processing in the primate cortex. Neuron 88:419-431. CrossRef Medline

Finelli LA, Baumann H, Borbély AA, Achermann P (2000) Dual electroencephalogram markers of human sleep homeostasis: correlation between theta activity in waking and slow-wave activity in sleep. Neuroscience 101:523-529. CrossRef Medline

Finelli LA, Achermann P, Borbély AA (2001) Individual 'fingerprints' in human sleep EEG topography. Neuropsychopharmacology 25:S57-S62. CrossRef Medline

Fisher SP, Cui N, McKillop LE, Gemignani J, Bannerman DM, Oliver PL, Peirson SN, Vyazovskiy VV (2016) Stereotypic wheel running decreases cortical activity in mice. Nat Commun 7:13138. CrossRef Medline

Freyer F, Roberts JA, Becker R, Robinson PA, Ritter P, Breakspear M (2011) Biophysical mechanisms of multistability in resting-state cortical rhythms. J Neurosci 31:6353-6361. CrossRef Medline

Friston K, Breakspear M, Deco G (2012) Perception and self-organized instability. Front Comput Neurosci 6:44. CrossRef Medline

Haldeman C, Beggs JM (2005) Critical branching captures activity in living neural networks and maximizes the number of metastable states. Phys Rev Lett 94:058101. CrossRef Medline

Honey CJ, Thesen T, Donner TH, Silbert LJ, Carlson CE, Devinsky O, Doyle WK, Rubin N, Heeger DJ, Hasson U (2012) Slow cortical dynamics and the accumulation of information over long timescales. Neuron 76:423434. CrossRef Medline

Ives AR (1995) Measuring resilience in stochastic systems. Ecol Monogr 65:217233.

Jones BE (2005) From waking to sleeping: neuronal and chemical substrates. Trends Pharmacol Sci 26:578-586. CrossRef Medline

Kiebel SJ, Daunizeau J, Friston KJ (2008) A hierarchy of time-scales and the brain. PLoS Comput Biol 4:e1000209. CrossRef Medline

Killgore WD (2010) Effects of sleep deprivation on cognition. Prog Brain Res 185:105-129. CrossRef Medline

Kringelbach ML, McIntosh AR, Ritter P, Jirsa VK, Deco G (2015) The rediscovery of slowness: exploring the timing of cognition. Trends Cogn Sci 19:616-628. CrossRef Medline

Linkenkaer-Hansen K, Nikouline VV, Palva JM, Ilmoniemi RJ (2001) Long-range temporal correlations and scaling behavior in human brain oscillations. J Neurosci 21:1370-1377. Medline

Lo JC, Groeger JA, Santhi N, Arbon EL, Lazar AS, Hasan S, von Schantz M, Archer SN, Dijk DJ (2012) Effects of partial and acute total sleep deprivation on performance across cognitive domains, individuals and circadian phase. PLoS One 7:e45987. CrossRef Medline

Markram H, et al. (2015) Reconstruction and Simulation of Neocortical Microcircuitry. Cell 163:456-492. CrossRef Medline

Massimini M, Ferrarelli F, Huber R, Esser SK, Singh H, Tononi G (2005) Breakdown of cortical effective connectivity during sleep. Science 309: 2228-2232. CrossRef Medline

McGinley MJ, Vinck M, Reimer J, Batista-Brito R, Zagha E, Cadwell CR, Tolias AS, Cardin JA, McCormick DA (2015) Waking state: rapid vari- ations modulate neural and behavioral responses. Neuron 87:1143-1161. CrossRef Medline

Meisel C, Storch A, Hallmeyer-Elgner S, Bullmore E, Gross T (2012) Failure of adaptive self-organized criticality during epileptic seizure attacks. PLoS Comput Biol 8:e1002312. CrossRef Medline

Meisel C, Olbrich E, Shriki O, Achermann P (2013) Fading signatures of critical brain dynamics during sustained wakefulness in humans. J Neurosci 33:17363-17372. CrossRef Medline

Meisel C, Klaus A, Kuehn C, Plenz D (2015) Critical slowing down governs the transition to neuron spiking. PLoS Comput Biol 11:e1004097. CrossRef Medline

Meisel C, Bailey K, Achermann P, Plenz D (2017) Decline of long-range temporal correlations during sustained wakefulness in the human brain. Sci Reports 7:11825. CrossRef

Mignot E (2008) Why we sleep: the temporal organization of recovery. PLoS Biol 6:e106. CrossRef Medline

Murray JD, Bernacchia A, Freedman DJ, Romo R, Wallis JD, Cai X, PadoaSchioppa C, Pasternak T, Seo H, Lee D, Wang XJ (2014) A hierarchy of intrinsic timescales across primate cortex. Nat Neurosci 17:1661-1663. CrossRef Medline

Nauhaus I, Busse L, Carandini M, Ringach DL (2009) Stimulus contrast modulates functional connectivity in visual cortex. Nat Neurosci 12:7076. CrossRef Medline

Nauhaus I, Busse L, Ringach DL, Carandini M (2012) Robustness of traveling waves in ongoing activity of visual cortex. J Neurosci 32:3088-3094. CrossRef Medline

Ogawa T, Komatsu H (2010) Differential temporal storage capacity in the baseline activity of neurons in macaque frontal eye field and area V4. J Neurophysiol 103:2433-2445. CrossRef Medline

Poulet JF, Petersen CC (2008) Internal brain state regulates membrane potential synchrony in barrel cortex of behaving mice. Nature 454:881-885. CrossRef Medline

Ray S, Maunsell JH (2011) Different origins of gamma rhythm and highgamma activity in macaque visual cortex. PLoS Biol 9:e1000610. CrossRef Medline

Ray S, Hsiao SS, Crone NE, Franaszczuk PJ, Niebur E (2008) Effect of stimulus intensity on the spike-local field potential relationship in the secondary somatosensory cortex. J Neurosci 28:7334-7343. CrossRef Medline

Scheffer M, Bascompte J, Brock WA, Brovkin V, Carpenter SR, Dakos V, Held H, van Nes EH, Rietkerk M, Sugihara G (2009) Early-warning signals for critical transitions. Nature 461:53-59. CrossRef Medline

Shew WL, Yang H, Petermann T, Roy R, Plenz D (2009) Neuronal avalanches imply maximum dynamic range in cortical networks at criticality. J Neurosci 29:15595-15600. CrossRef Medline

Shoham S, Fellows MR, Normann RA (2003) Robust, automatic spike sorting using mixtures of multivariate t-distributions. J Neurosci Methods 127:111-122. CrossRef Medline

Stephens GJ, Honey CJ, Hasson U (2013) A place for time: the spatiotemporal structure of neural dynamics during natural audition. J Neurophysiol 110:2019-2026. CrossRef Medline

Strijkstra AM, Beersma DG, Drayer B, Halbesma N, Daan S (2003) Subjective sleepiness correlates negatively with global alpha $(8-12 \mathrm{~Hz})$ and positively with frontal theta $(4-8 \mathrm{~Hz})$ frequencies in the human resting awake electroencenphalogram. Neurosci Lett 340:17-20. CrossRef Medline

Tagliazucchi E, von Wegner F, Morzelewski A, Brodbeck V, Jahnke K, Laufs H (2013) Breakdown of long-range temporal dependence in default mode and attention networks during deep sleep. Proc Natl Acad Sci U S A 110: 15419-15424. CrossRef Medline

Tononi G (2008) Consciousness as integrated information: a provisional manifesto. Biol Bull 215:216-242. CrossRef Medline

Van Dongen HP, Maislin G, Mullington JM, Dinges DF (2003) The cumulative cost of additional wakefulness: dose-response effects on neurobehavioral functions and sleep physiology from chronic sleep restriction and total sleep deprivation. Sleep 26:117-126. CrossRef Medline

Vyazovskiy VV, Olcese U, Hanlon EC, Nir Y, Cirelli C, Tononi G (2011) Local sleep in awake rats. Nature 472:443-447. CrossRef Medline

Werth E, Achermann P, Borbély AA (1997) Fronto-occipital EEG power gradients in human sleep. J Sleep Res 6:102-112. CrossRef Medline

Wissel C (1984) A universal law of the characteristic return time near thresholds. Oecologia 65:101-107. CrossRef Medline 